\title{
Structure of the Particle-Hole Amplitudes in No-core Shell Model Wave Functions
}

\author{
A.C. Hayes ${ }^{1}$, A.A. Kwiatkowski ${ }^{2}$ \\ ${ }^{1}$ Theoretical Division, Los Alamos National Laboratory, \\ Los Alamos, New Mexico 87545 \\ ${ }^{2}$ National Superconducting Cyclotron Laboratory, \\ Michigan State University, \\ East Lansing, Michigan 48824
}

\begin{abstract}
We study the structure of the no-core shell model wave functions for ${ }^{6} \mathrm{Li}$ and ${ }^{12} \mathrm{C}$ by investigating the ground state and first excited state electron scattering charge form factors. In both nuclei, large particle-hole $(p h)$ amplitudes in the wave functions appear with the opposite sign to that needed to reproduce the shape of the $\left(e, e^{\prime}\right)$ form factors, the charge radii, and the $\mathrm{B}(\mathrm{E} 2)$ values for the lowest two states. The difference in sign appears to arise mainly from the monopole $\Delta \hbar \omega=2$ matrix elements of the kinetic and potential energy $(\mathrm{T}+\mathrm{V})$ that transform under the harmonic oscillator $\mathrm{SU}(3)$ symmetries as $(\lambda, \mu)=(2,0)$. These are difficult to determine self-consistently, but they have a strong effect on the structure of the low-lying states and on the giant monopole and quadrupole resonances. The Lee-Suzuki transformation, used to account for the restricted nature of the space in terms of an effective interaction, introduces large higher-order $\Delta \hbar \omega=n, n>2, p h$ amplitudes in the wave functions. The latter $p h$ excitations aggravate the disagreement between the experimental and predicted $\left(e, e^{\prime}\right)$ form factors with increasing model spaces, especially at high momentum transfers. For sufficiently large model spaces the situation begins to resolve itself for ${ }^{6} \mathrm{Li}$, but the convergence is slow. A prescription to constrain the $p h$ excitations would likely accelerate convergence of the calculations.
\end{abstract}

PACS numbers: 21.60.-n,21.60.Cs,21.60.De,24.30.Cz,25.30.Bf,25.30.Dh

\section{INTRODUCTION}

The ab initio no-core shell model (NCSM) permits calculations of wave functions in very large model-space sizes for nuclei at the beginning of the p-shell. For ${ }^{6} \mathrm{Li}$ calculations up to $16 \hbar \omega$ have been achieved [1]. Among the successes of the model is its predicted energy spectra of light nuclei [1, 2, 3]. Towards the end of the p-shell a $10 \hbar \omega$ basis calculation has been achieved for $A=11$ [4]. In mass 12 the model provides a reasonable description of the low-momentum component of the vector and axial currents involved in the electro-weak transitions to the ground state on ${ }^{12} \mathrm{~N}$ when a three-body interaction is included [6]. In all of these calculations, a Lee-Suzuki [5] transformation of the nucleon-nucleon interaction is used to account for the restricted nature of the space in terms of an effective interaction. Group theoretical analyses [7] of the no-core shell model wave functions have shown that the predicted eigenstates of ${ }^{12} \mathrm{C}$ and ${ }^{16} \mathrm{O}$ have very large overlaps with a small sub-space of the full model space, with the sub-space being defined by the most deformed symplectic basis states. The purpose of this paper is to examine the structure of the multi- $\hbar \omega$ terms in the wave functions for the low-lying states at the beginning and end of the $p$-shell in more detail. For this we compare NCSM predictions with measured elastic and inelastic $\left(e, e^{\prime}\right)$ charge form factors in ${ }^{6} \mathrm{Li}$ and ${ }^{12} \mathrm{C}$.

The shape of the electron scattering form factors provides a direct probe of the magnitude and structure of the higher shell components in the wave functions. The charge form factors have the additional advantage that two-body meson-exchange currents do not contribute significantly to the form factors below about $2 \mathrm{fm}^{-1}[8]$. In all $\left(e, e^{\prime}\right)$ calculations presented here we use a bare onebody operator; as discussed below, the introduction of an effective operator (to compensate for the truncated model space) does not significantly affect our conclusions.

Our first main finding is that the $p h$ amplitudes that contribute significantly to electron scattering appear with the opposite sign to that needed to replicate the experimental form factors, elastic and inelastic, and the charge radii. The $2 \hbar \omega$ contributions to the inelastic form factor change sign, in agreement with experiment, for sufficiently large model spaces for ${ }^{6} \mathrm{Li}$; however, higher-order terms do not within the model spaces we examined. Second we show that the symplectic $(\lambda, \mu), \Delta \hbar \omega=2 p h$ amplitudes in the wave functions are sensitive functions of the oscillator parameter.

\section{THE ELASTIC CO FORM FACTORS}

The ground state $\mathrm{C} 0$ form factor is the Fourier transform of the charge density, and contributions from twobody charge operators and/or relativistic corrections are negligible for momenta up to about $2 \mathrm{fm}^{-1}[8]$. In a harmonic oscillator (HO) basis, the $0 \hbar \omega$-shell charge form factor is given by

$$
F_{0 p-0 p}\left(q^{2}\right)=\sqrt{3}(1-2 / 3 y) \exp (-y)
$$

where $y=(b q / 2)^{2}$ and $b$ is the oscillator parameter.

When additional shells are added to the model space the new contributions to the form factor fall into two 
main classes. The first of these are the in-shell contributions (e.g. $1 s 0 d-1 s 0 d, 1 p 0 f-1 p 0 f$, etc.) determined by the occupation numbers for the higher shells, and the second are from cross-shell ph excitations (e.g. $0 s-1 s, 0 p-1 p, 0 s-2 s$, etc.). At low $q$ the form factor is determined by the charge radius

$$
F\left(q^{2}\right)=1-\frac{<r^{2}>q^{2}}{6}+O\left(q^{4}\right)
$$

and the higher in-shell contributions can be shown always to add constructively to the charge radius. The cross-shell excitations (which for HO wave functions contribute to the charge radius only for $\Delta \hbar \omega=2 p h$ excitations across two shells) can add constructively or destructively. In a $2 \hbar \omega$ calculation for p-shell nuclei there are two possible cross-shell contributions, namely,

$$
\begin{gathered}
F_{0 s-1 s}\left(q^{2}\right)=\sqrt{2 / 3} y \exp (-y) \\
F_{0 p-1 p}\left(q^{2}\right)=\sqrt{10 / 3} y(1-2 / 5 y) \exp (-y)
\end{gathered}
$$

Both of these transform under $\mathrm{SU}(3)$ as $(\lambda, \mu)=(2,0)$ and represent the $2 \hbar \omega$ symplectic contributions to the form factors. When these and/or higher shell $\Delta \hbar \omega=$ $2 p h$ excitations appear with a sign so as to enhance the predicted charge radius, they pull in the charge form factor in momentum space.

\section{A. C0 form factor for ${ }^{6} \mathrm{Li}$}

Both elastic and inelastic scattering from ${ }^{6} \mathrm{Li}$ have been studied extensively [9, 10, 11, 12, 13, 14]. Our calculations use the CD Bonn nucleon-nucleon interaction [15]. The predicted ground state energy of ${ }^{6} \mathrm{Li}$ is least sensitive to the choice of the oscillator parameter $b$ over increasing basis size for $b=1.79 \mathrm{fm}(\hbar \omega=13 \mathrm{MeV})$ [1], which is the value we use in the present $\left(\mathrm{e}, \mathrm{e}^{\prime}\right)$ calculations. We note that, because of the increased computational difficulties in calculating the transition density matrix elements needed for the (e,e') form factors, the present calculations are restricted to a maximum model space of $14 \hbar \omega$, to be compared with the $16 \hbar \omega$ model spaces used to calculate the energy spectra for ${ }^{6} \mathrm{Li}$.

Figure 1 compares the experimental data for the elastic form factor for ${ }^{6} \mathrm{Li}$ with the model predictions. For model spaces up to $10 \hbar \omega$, the predicted form factor moves out further in momentum space as the basis is increased. For the largest model space examined, $14 \hbar \omega$, the tend begins to reverse. In coordinate space the predicted charge density (Figure 2) is enhanced in the interior, with little change to the tail as higher shells are added. These trends reflect the structure and sign of $p h$ excitations introduced as the model space increases. There are two issues with the structure of the predicted ground state

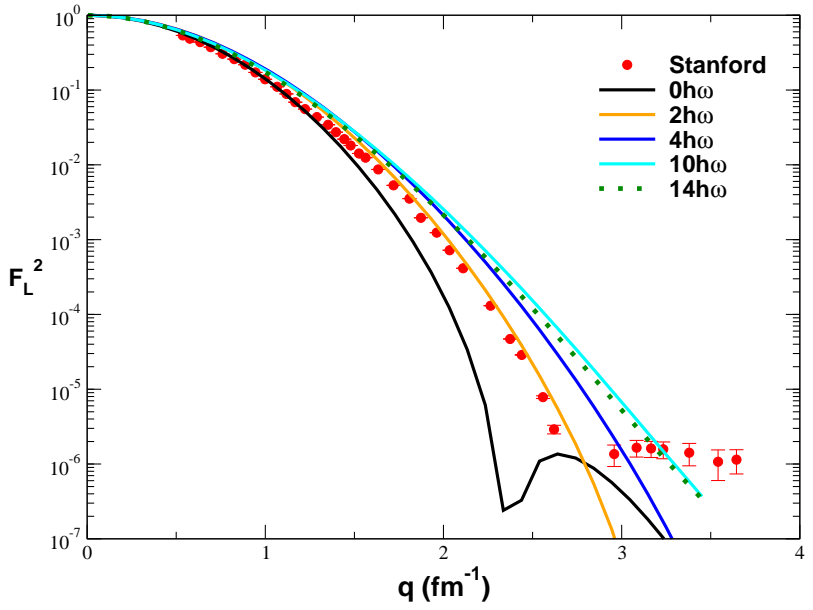

FIG. 1: (color online)The elastic C0 form factor for the ground state of ${ }^{6} \mathrm{Li}$. For all but the largest model space, the form factor moves further out in $q$ as the model space is increased. Experimental data are taken from [12]

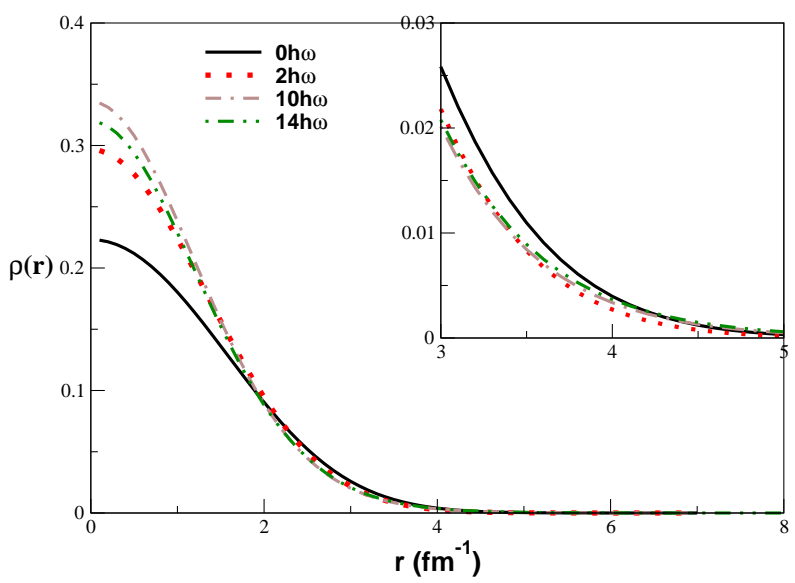

FIG. 2: (color online) The ground state charge density of ${ }^{6} \mathrm{Li}$. As the model space increases the density is enhanced in the interior, with minor changes in the tail region. For sufficiently large model spaces the strength in the interior starts to become suppressed with a corresponding build up in the tail region.

$p h$ excitations. First, the $\Delta \hbar \omega=2 p h$ excitation for all shells included add destructively to the ground state charge radius. These suggest that the sign of the important symplectic excitations in the wave functions may be problematic. In momentum-space these excitations pull the form factor out in $q$. Second, there are large $0 s \rightarrow n s$ excitations which pull the form factor out further at higher $q$. These effects are shown in Figures 3 


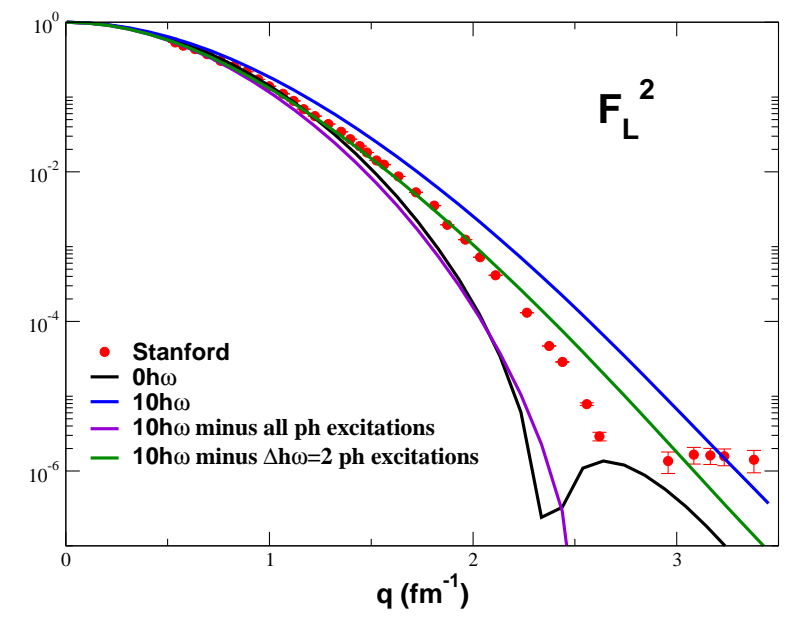

FIG. 3: color online) The ground state C0 form factor for ${ }^{6} \mathrm{Li}$. The figure displays the effect of particle-hole excitations on the predicted form factor by arbitrarily setting the one-body density matrix elements to zero. Experimental data are taken from [12].

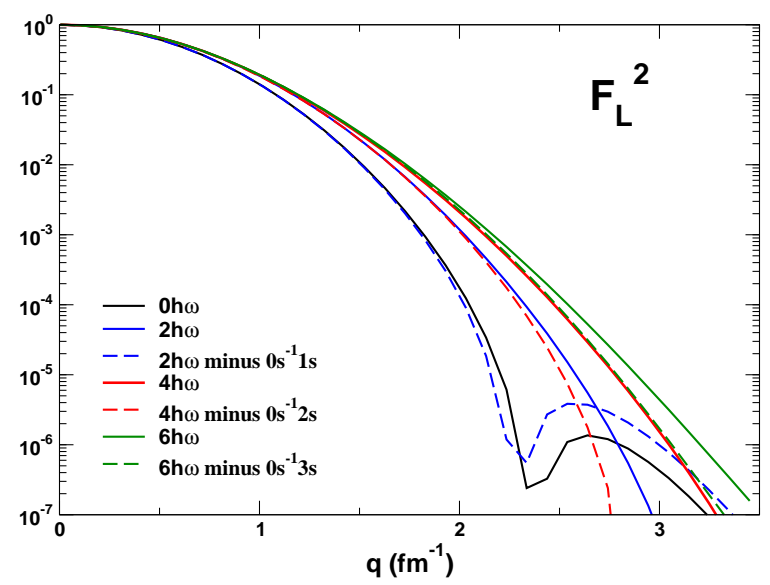

FIG. 4: (color online) The ground state $\mathrm{C} 0$ form factor for ${ }^{6} \mathrm{Li}$. The figure displays the effect of $0 s \rightarrow n s$ ph excitations on the predicted form factor. The dashed curves show the effect of setting the one-body density matrix elements for these excitation to zero (arbitrarily). The $0 s \rightarrow n s$ excitations are sizable and pull the form factor out further at higher momentum transfers.

and 4 , where the relevant $p h$ one-body density matrix elements have been arbitrarily set to zero for the purposes of displaying their effect on the shape of the predicted form factor.

In Table 1 we show the contributions to the charge radius from in-shell versus cross-shell excitations, and the destructive interference from the $p h$ excitations is larger

\begin{tabular}{|c|cccccc|}
\hline \multicolumn{6}{|c|}{ Point charge radius for ${ }^{6} \mathrm{Li}$ in units of fm } \\
\hline Model space & $0 \hbar \omega$ & $2 \hbar \omega$ & $4 \hbar \omega$ & $10 \hbar \omega$ & $14 \hbar \omega$ & Expt. \\
\hline full model space & 2.23 & 2.08 & 2.1 & 2.14 & 2.21 & $2.38 \pm 0.1$ \\
$p h$ contributions omitted & 2.23 & 2.26 & 2.31 & 2.38 & 2.41 & \\
\hline
\end{tabular}

TABLE I:

than the constructive interference from the higher in-shell excitations. This suggests that the predicted sign of the $\Delta \hbar \omega=2 p h$ excitations that transform under $\mathrm{SU}(3)$ as $(\lambda, \mu)=(2,0)$ inhibits the convergence of the calculations.

\section{B. Co form factor for ${ }^{12} \mathrm{C}$}

The trends seen for the elastic scattering form factor for ${ }^{6} \mathrm{Li}$ are also seen in the case of ${ }^{12} \mathrm{C}$. Again the calculations use the CD Bonn interaction [15]. The $\Delta \hbar \omega=2$ $p h$ excitations add destructively to the charge radius and pull the elastic $\mathrm{C} 0$ form factor out in momentum space, Figure 5. We note that the charge radius for ${ }^{12} \mathrm{C}$ (Table III) is over-predicted, which in part reflects the chosen oscillator parameter, $b=1.663 \mathrm{fm}(\hbar \omega=15 \mathrm{MeV})$, which minimizes the ground state energy. We will discuss the choice and effect of $b$ in section $\mathrm{V}$. As in the case of ${ }^{6} \mathrm{Li}$,

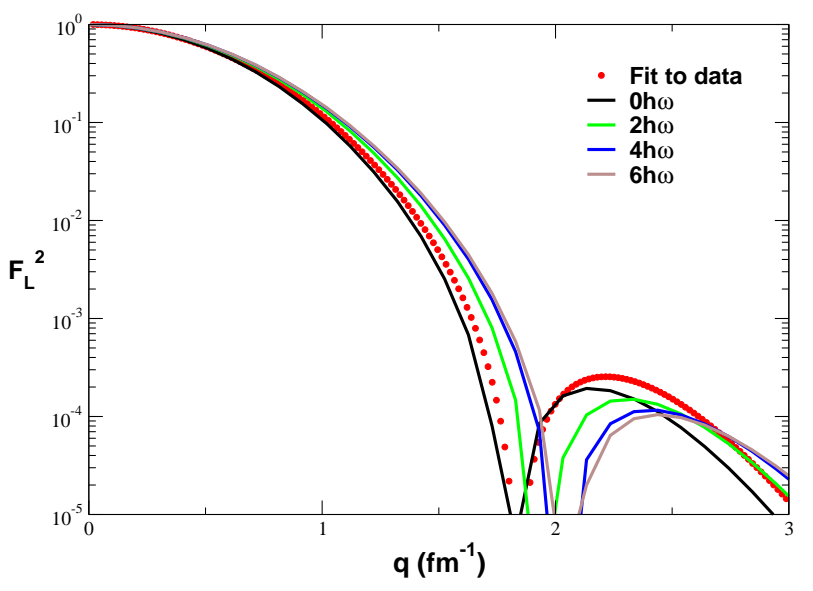

FIG. 5: (color online) The elastic C0 form factor for the ground state of ${ }^{12} \mathrm{C}$. As the model space increases the form factor moves further out in $q$, and the dominant higher shell terms add destructively to the predicted charge radius. Experimental data were taken from [17, 18, 19, 20].

ph excitations across more than two shells are large, and they act so as to move the form factor out further in $q$. For ${ }^{12} \mathrm{C}$ these include both the $0 s \rightarrow n s$ and $0 p \rightarrow n p$ excitations. Figure [6 displays the effect of arbitrarily setting the $p h$ contributions to the form factor to zero. 


\begin{tabular}{|c|lllll|}
\hline \multicolumn{4}{|c|}{ Point charge radius for ${ }^{12} \mathrm{C}$ in units of fm } \\
\hline Model space & $0 \hbar \omega$ & $2 \hbar \omega$ & $4 \hbar \omega$ & $6 \hbar \omega$ & Expt. \\
\hline full model space & 3.18 & 2.99 & 2.95 & 2.95 & $2.32 \pm 0.022$ \\
$p h$ contributions omitted & 3.18 & 3.20 & 3.25 & 3.24 & \\
\hline
\end{tabular}

TABLE II:

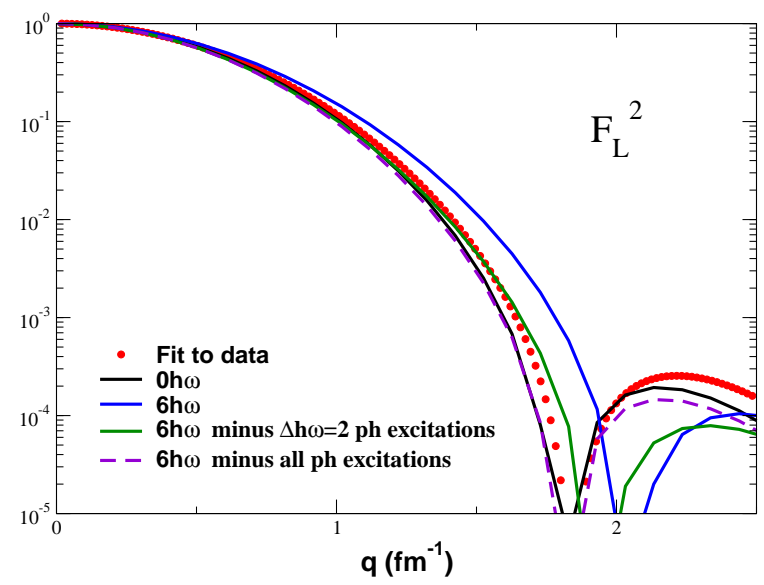

FIG. 6: (color online) The elastic C0 form factor for the ground state of ${ }^{12} \mathrm{C}$. The $6 \hbar \omega$ calculations displaying the effect of the removal of the $p h$ excitations were obtained by arbitrarily setting the corresponding one-body density matrix elements to zero.

\section{C2 FORM FACTORS}

The $\mathrm{C} 2$ form factor is determined by the transition charge density; there is no significant contribution from two-body meson exchange currents below $q \approx 2 \mathrm{fm}^{-1}[16]$. We examine the longitudinal form factor for scattering to the $2.186 \mathrm{MeV}\left(3^{+} \mathrm{T}=0\right)$ state in ${ }^{6} \mathrm{Li}$ and the $4.44 \mathrm{MeV}$ $\left(2^{+} \mathrm{T}=0\right)$ state in ${ }^{12} \mathrm{C}$. Data for the ${ }^{6} \mathrm{Li} \mathrm{C} 2$ transition have been measured to $q \approx 3.5 \mathrm{fm}^{-1}[12,13$, 14]. Extensive data are also available for the $4.44 \mathrm{MeV}\left(2^{+}\right)$state in ${ }^{12} \mathrm{C}$ [17, 18, 19, 20].

The most significant contributions to the $\mathrm{C} 2$ form factors for $p$-shell nuclei in a $(0+2) \hbar \omega$ calculation are transitions within the $p$-shell $(0 p \rightarrow 0 p)$ and $p h$ excitations across two shells that correspond to the excitation of the GQR. The latter transform under $\mathrm{SU}(3)$ as $(\lambda, \mu) L=(2,0) 2$. For $\mathrm{HO}$ wave functions the $0 p \rightarrow 0 p$ and GQR form factors are 21]

$$
\begin{gathered}
F_{p-p}\left(q^{2}\right)=-\sqrt{8 / 15} y \exp (-y) \\
F_{G Q R}\left(q^{2}\right)=\sqrt{24 / 15} y(1-1 / 3 y) \exp (-y),
\end{gathered}
$$

and as before $y=(b q / 2)^{2}$. If a small admixture of the GQR is added to the $0 \hbar \omega$ state so as to enhance the

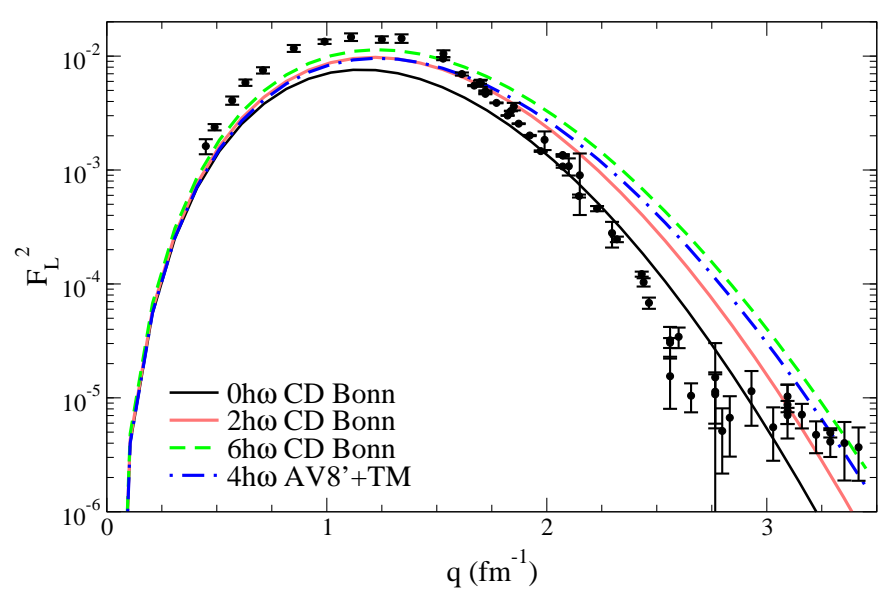

FIG. 7: (color online) The longitudinal electron scattering form factor for the $4.44 \mathrm{MeV}\left(2^{+} \mathrm{T}=0\right)$ state in ${ }^{12} \mathrm{C}$. As the model space increases the form factor moves further out in $q$, in contradiction with experimental and theoretical expectations.

$\mathrm{B}(\mathrm{E} 2)$, the form factor is suppressed at high $q$. For larger model spaces higher powers of $y$ are introduced.

The shape of the predicted $\mathrm{F}_{L}$ is often displayed in terms of the $\mathrm{C} 2$ matrix element 21]. In general, the $\mathrm{C} \lambda$ matrix element is defined in terms of the form factor $F_{\lambda}$ as,

$$
B(C \lambda)=f^{-2} \frac{Z^{2}}{4 \pi}\left(\frac{(2 \lambda+1) ! !}{q^{\lambda}}\right)^{2} F_{\lambda}^{2}
$$

and

$$
C 2(q) \equiv B(C 2)^{1 / 2}=A+B y+C y^{2}+\ldots
$$

where $f=f_{S N} f_{c . m .} \exp (-y), f_{S N}$ is the single-nucleon charge form factor [22] and the center of mass correction is $f_{c . m}=\exp (y / A)$ [23]. For a HO basis, the number of terms appearing in the polynomial (6) is determined by the number of shells included in the calculation. For $p$ shell nuclei the experimental $\mathrm{C} 2(q)$ matrix elements for low-lying states generally decreases with increasing $q^{2}$, i.e., the coefficient ratio $A / B<0$, where $A>0$.

\section{A. C2 Form Factor in ${ }^{12} \mathrm{C}$}

Our calculations for ${ }^{12} \mathrm{C}$ include model spaces up to $6 \hbar \omega$. We use the CD Bonn [15] and the AV8' [24] nucleonnucleon interactions, as well as the AV8' plus the TucsonMelbourne TM'(99) 3-body [25] interactions. The oscillator parameter was taken to be $b=1.663 \mathrm{fm} \quad(\hbar \omega=15$ $\mathrm{MeV})$.

Figure 7 shows a comparison between the measured and predicted form factors for increasing sizes of the shell 


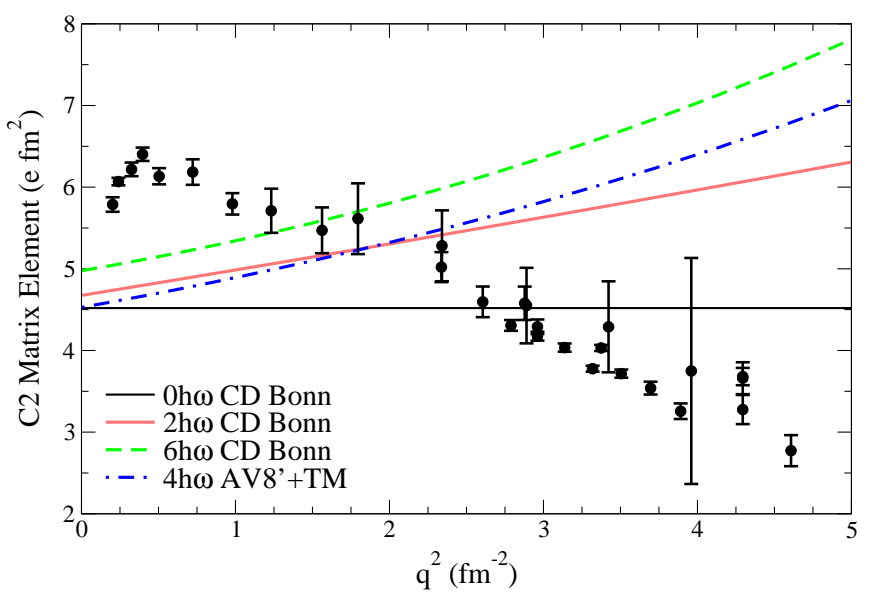

FIG. 8: (color online) The C2 matrix element for the 4.44 $\mathrm{MeV}\left(2^{+} \mathrm{T}=0\right)$ state in ${ }^{12} \mathrm{C}$. The effect of using a threebody interaction (AV8'+TM) is small. Experimental data were taken from [17, 18, 19, 20]

model space. At low momentum transfers the calculations under-predict the form factor. Above $q \approx 1.5 \mathrm{fm}^{-1}$ the calculations over-predict the form factor, and this over-prediction becomes increasingly worse as the size of the model space is increased. As the model space is increased beyond $0 \hbar \omega$, the $0 p \rightarrow 1 p$ excitations add destructively at low $q$ (and thus destructively to the the $\mathrm{B}(\mathrm{E} 2)$ value) and constructively at high $q$, moving the form factor out. The predicted form factor is enhanced slightly at small $q$ relative to the $0 \hbar \omega$ calculation, and we largely attribute this to the $0 s \rightarrow 1 d$ excitations, which appear with the correct sign. We note this differs from the elastic $\mathrm{C} 0$ form factors where excitations from the $0 \mathrm{~s}$ shell appear with the same sign as excitations from the $0 p$ shell. It should be noted that the model spaces examined here are restricted to $6 \hbar \omega$ and that much larger spaces may well show very different trends.

Figure 8 displays $\mathrm{C} 2(q)$, which we extracted from the measured form factor using $b=1.7 \mathrm{fm}$. The experimental C2 $(q)$ matrix element steadily drops with increasing $q^{2}$. Our multi- $\hbar \omega$ calculations predict $\mathrm{C} 2(q)$ to have the opposite slope, in large part because of the sign of the $(0 p)^{-1}(1 p)$ excitations in the $0^{+}$and $2^{+}$wave functions.

Figure 9 displays the corresponding transition charge density $\rho(\mathrm{r})$ for the $0^{+} \rightarrow 2^{+}$transition. The experimentally determined $\rho(r)$ peaks at about $2 \mathrm{fm}$, while the $\rho(r)$ predicted by the NCSM peaks at about $1.5 \mathrm{fm}$. As the model space is increased, the peak moves towards smaller $r$.

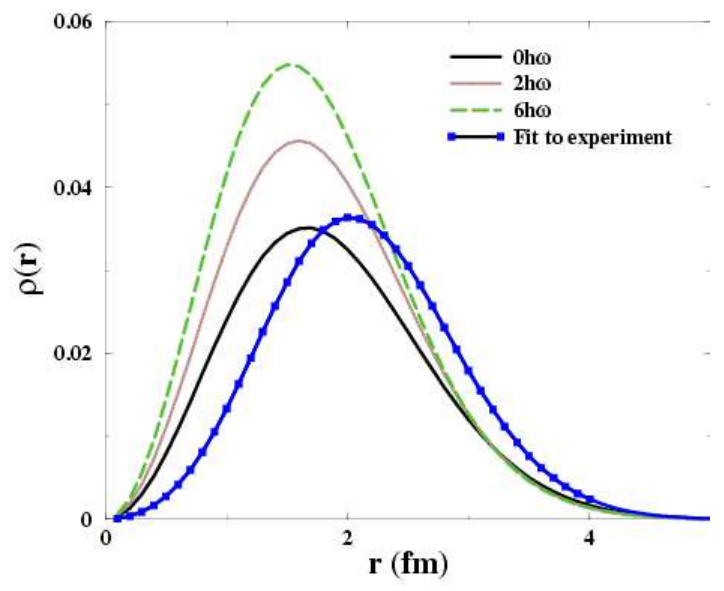

FIG. 9: (color online) The transition charge density for the $4.44 \mathrm{MeV}\left(2^{+} \mathrm{T}=0\right)$ state in ${ }^{12} \mathrm{C}$. As the model space is increased the transition density moves towards smaller $r$.

\section{B. The C2 form factor for ${ }^{6} \mathrm{Li}$}

Figure 10 displays a comparison of the measured and predicted form factors for increasing basis size for $b=1.79 \mathrm{fm}$. These calculations use the CD Bonn nucleon-nucleon interaction [15]. At low momentum transfers, $q<1.0 \mathrm{fm}^{-1}$, the calculations under-predict the form factor. At $q>1.5 \mathrm{fm}^{-1}$, the larger model spaces over-predict the magnitude of the form factor, and this over-prediction increases with the model space size. The general trends seen with the sign of higher shell contri-

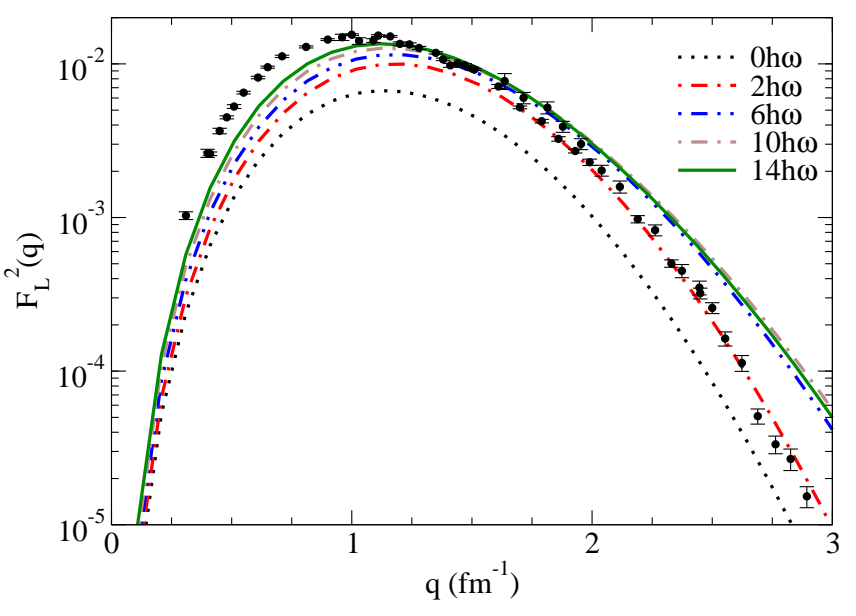

FIG. 10: (Color online) The charge form factor for the $3^{+}$ $2.186 \mathrm{MeV}$ state using ab initio NCSM CD Bonn wave functions, where $b=1.79 \mathrm{fm}$. At this value of $b(\hbar \omega=13 \mathrm{MeV})$, the ground state energy converges fastest over basis size. Empirical data are taken from Stanford, Saskatoon, and Mainz [12, 13, 14]. 


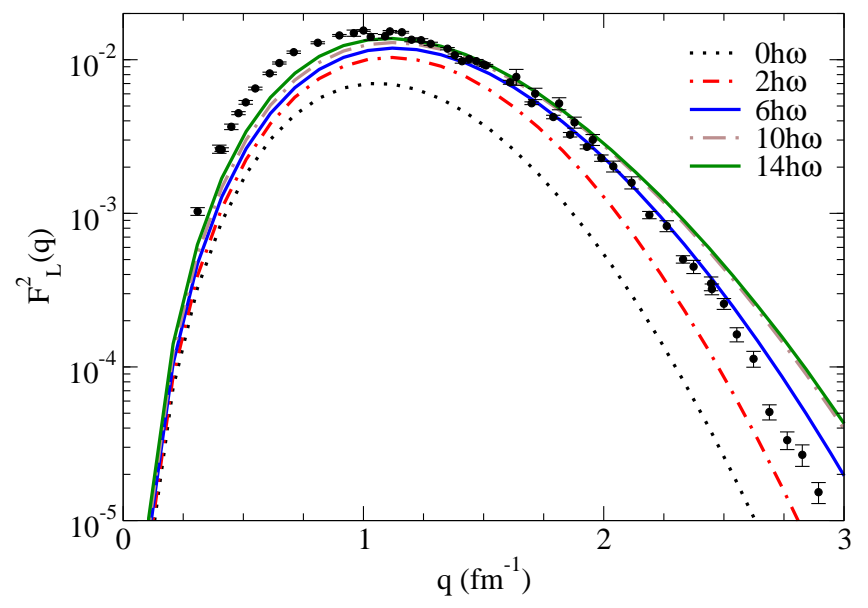

FIG. 11: (Color online) The charge form factor is calculated with $b=1.94 \mathrm{fm}(\hbar \omega=11 \mathrm{MeV})$ for comparison to figure 10 The predictions are suppressed at low $q$ and enhanced at high $q$ for all size model spaces. Experimental data are taken from [12, 13, 14]

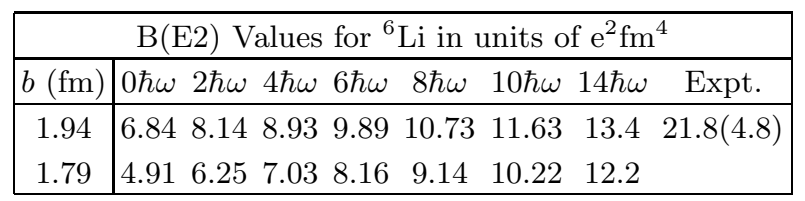

TABLE III:

butions in the predicted $\mathrm{C} 2$ form factors are very similar to those seen for ${ }^{12} \mathrm{C}$. However, our ability to go to considerably higher shells in the case of ${ }^{6} \mathrm{Li}$ allows us to explore these trends in more detail. The peak of the predicted form factor occurs at higher $q$ than experiment; the biggest shift of the predicted form factor away from the observed peak occurs between the $0 \hbar \omega$ and the $2 \hbar \omega$ model space, and a shift in the peak position to even higher $q$ continues until $8 \hbar \omega$. Above $8 \hbar \omega$ the peak position of the form factor begins to improve. Above the peak, at momenta $q>1.3 \mathrm{fm}^{-1}$, the additional contributions from higher shell continue to enhance the form factor. For low momentum transfers (below the peak of the form factor) we see a slow convergence to a magnitude lower than experiment. This low momentum trend is consistent with the trend of predicted $\mathrm{B}(\mathrm{E} 2)$ values, as summarized in Table III.

We also examined the form factor for a set of calculations with a different oscillator parameter, namely, $b$ $=1.94 \mathrm{fm}(\hbar \omega=11 \mathrm{MeV})$, Figure 11. The $b=1.94 \mathrm{fm}$ form factors display similar qualitative behavior as the $b$ $=1.79 \mathrm{fm}$ calculations. The peak of the former occurs at higher $q$ than experiment and continues to shift outward until about $10 \hbar \omega$. For large model spaces the situation starts to improve. Above the peak of the form factor the

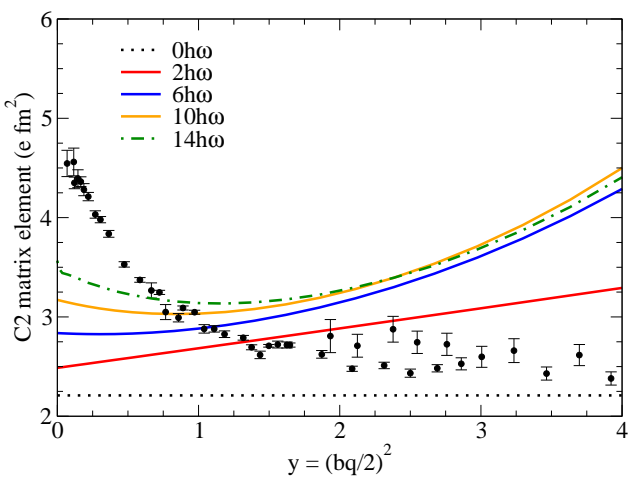

FIG. 12: (Color online) The C2 matrix element with $b=1.79$ $\mathrm{fm}$. To calculate the empirical $\mathrm{C} 2$ matrix element (data from $[12,13,14])$, we used $b=1.70 \mathrm{fm}$.

higher shell contributions move the form factor further out in $q$ with increasing model space.

The enhancements for the form factors at large $q$ are determined by the sign and magnitude of the higher shell contributions in the wave functions. As in the case of ${ }^{12} \mathrm{C}$, this is most striking in the case of the $2 \hbar \omega$ configurations, where the $0 p \rightarrow 1 p 0 f$ ph excitations add destructively at low $q$ and constructively at high $q$. The slow convergence of the $\mathrm{B}(\mathrm{E} 2)$ to a value smaller than experiment is due in large part to the fact that these $p h$ excitations add destructively to the matrix element.

A more detailed understanding of the convergence of the lower momentum terms in the form factor with increasing model space can be seen by examining the $\mathrm{C} 2$ matrix element. To obtain an experimental $\mathrm{C} 2$ matrix element, we chose an oscillator parameter $b=1.70 \mathrm{fm}$, which is close to the value necessary to give the measured rms charge radius. The $\mathrm{C} 2$ calculated matrix elements are displayed in Figures 12 and 13 . The experimental C2 matrix element decreases with increasing momentum transfer in contrast to the predictions of the model.

We graph the ratio of the coefficients $B / A$ appearing in eq. 6 in Figure 14 as a function of basis size. For the smaller model spaces this ratio has the wrong sign, but as the model space increases the sign eventually changes in qualitative agreement with experiment. For $b=1.79$ $\mathrm{fm}$, the ratio changes sign between $4 \hbar \omega$ and $6 \hbar \omega$ model spaces; for the $b=1.94 \mathrm{fm}$, the sign switches between $8 \hbar \omega$ and $10 \hbar \omega$.

Except for the largest model space examined, we see an approximate linear relationship between basis size and 


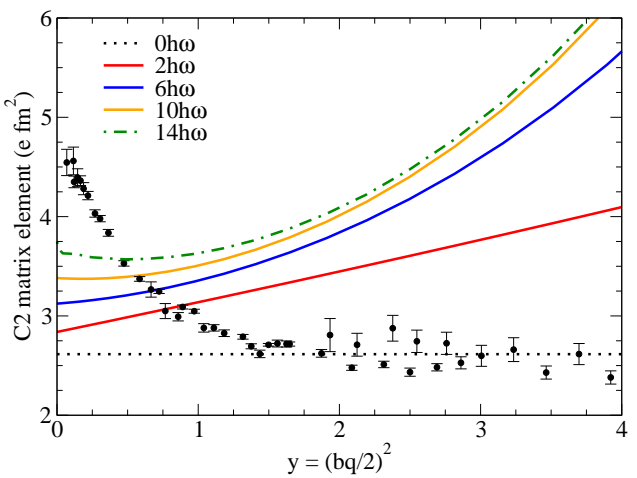

FIG. 13: (Color online) The C2 matrix element with $b=1.94$ fm. Experimental data are taken from [12, 13, 14] and a C2 matrix element extracted usine $b=1.70 \mathrm{fm}$.

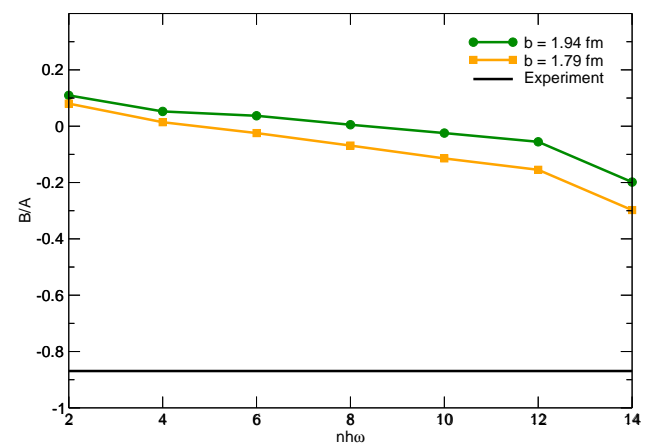

FIG. 14: (Color online) We fitted the C2 matrix element to the polynomial $A+B y+C y^{2} \ldots$ The ratio of $B / A$ is graphed along the $y$-axis. Since $A$ is always positive, the change in sign is exclusively in $B$. Experimental data was taken from [12, 13, 14] and a $B / A$ ratio was extracted using $b=1.70 \mathrm{fm}$.

$B / A$ and $C / A$, Figs. 14]15] The $14 \hbar \omega$ model space calculation suggests that the rate of convergence starts to increaco foctar than thic linaser ralatinnchin curmact

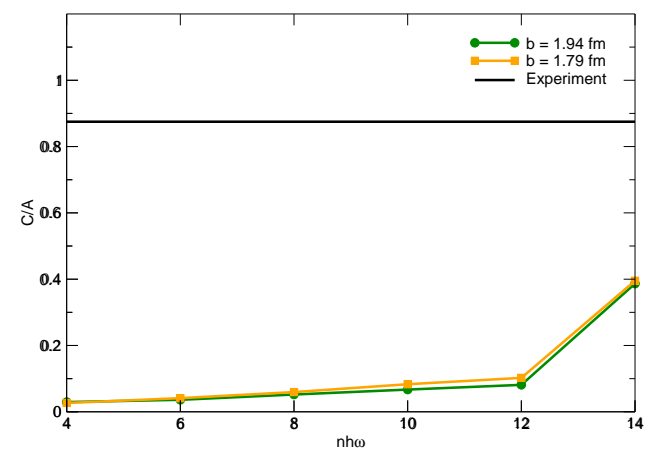

FIG. 15: (Color online) The ratio $C / A$ (eq. (3)) from a fit to the $\mathrm{C} 2$ matrix element in ${ }^{6} \mathrm{Li}$ as a function of the model space. Experimental data are taken from [12, 13, 14], and a $C / A$ ratio was extracted using $b=1.70 \mathrm{fm}$.

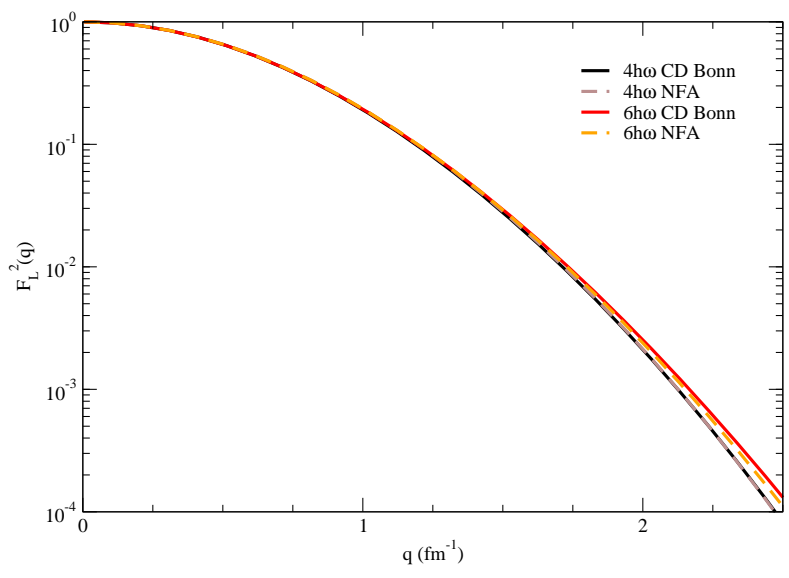

FIG. 16: (color online) Comparison of the predicted elastic $\mathrm{C} 0$ form factor for the ground state of ${ }^{6} \mathrm{Li}$ with and without the inclusion of a 3-body interaction.

\section{INCLUSION OF A 3-BODY INTERACTION}

The inclusion of a 3-body interaction leads to an improved predicted level spectrum in ${ }^{6} \mathrm{Li}$, particularly for the splitting between the ground state and the first $3^{+}$ state. In addition, the magnetic form factor for the $0^{+} \rightarrow 1^{+}$transition in ${ }^{12} \mathrm{C}$ is significantly improved when a 3-body interaction is included [6]. This is because the predicted form factor is very sensitive to the strength of the spin-orbit interaction. However, the present $(\Delta L=\lambda$ $\Delta S=0) C \lambda$ charge form factors are largely insensitive to the strength of the spin-orbit interaction and consequently to the 3-body interaction. In Figure [16] we compare the predicted $4 \hbar \omega$ and $6 \hbar \omega$ predictions for the elastic $\mathrm{C} 0$ form factor for ${ }^{6} \mathrm{Li}$. Figure 17 shows the equivalent calculations for the transition $\mathrm{C} 2$ form factor to the $3^{+}$ state. In both cases the inclusion of the 3-body interaction has little effect on the predicted form factor although it does improve the shape at higher $q$ very slightly. Figure 7 shows the effect of the 3-body interaction for the inelastic $\mathrm{C} 2$ form factor of ${ }^{12} \mathrm{C}$, which is also very small.

\section{DEPENDENCE ON THE OSCILLATOR PARAMETER}

The unexpected sign for some of the higher shell components in our NCSM calculations bears strong resemblance to a similar problem found in standard multi- $\hbar \omega$ HO shell model calculations. When HO standard shell model calculations are extended to include multi- $\hbar \omega$ configurations the lack of self-consistency (in the HartreeFock sense) causes some of the higher shell components in the wave functions to be unphysical [26, 27, 28, 29, 30, 

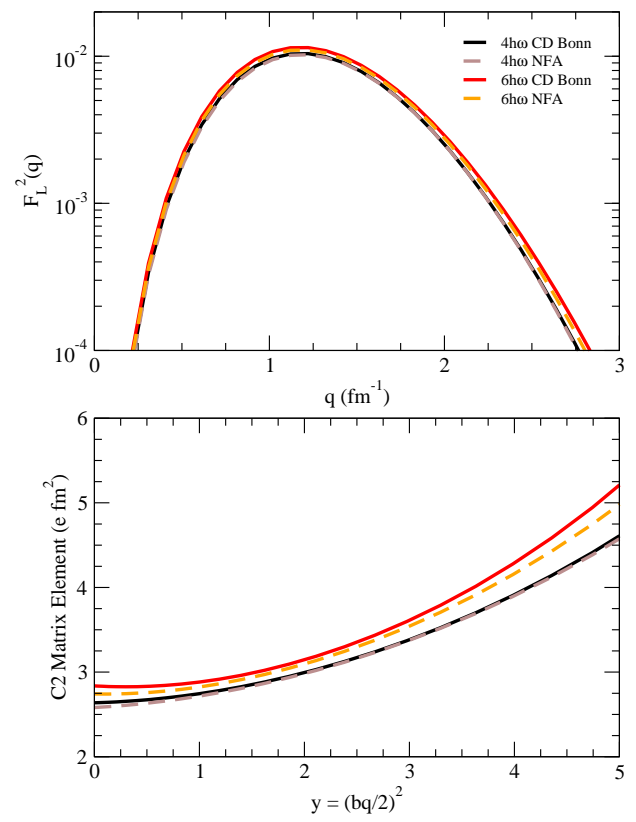

FIG. 17: (color online) Comparison of the predicted C2 form factor for the $3^{+}$state of ${ }^{6} \mathrm{Li}$ with and without the inclusion of a 3-body interaction.

31, 32, 33]. The main problem arises because matrix elements of the kinetic energy $(T)$ and the two-body interaction $(V)$ across two shells $(\Delta \hbar \omega=2)$ are large and opposite in sign, and they cannot be calculated reliably. The dependence of the $\mathrm{C} 2$ matrix element on $b$ implies that the magnitude and even the sign of the $\langle p h|T+V| 0 \hbar \omega\rangle$ matrix elements depend on $b$. These off-diagonal matrix elements across two shells in turn affect the sign of the leading $p h$ excitations in the wave functions, as well as all of the similar $\Delta \hbar \omega=2$ matrix elements up to the maximum shell included in the calculation.

\section{A. Dependence of ${ }^{6} \mathrm{Li}$ Form factors on the Oscillator Parameter}

We investigated the effect of the oscillator parameter on the predicted form factor within the $2 \hbar \omega$ model space, using four different values of $b$ ranging from $b=1.66$ $1.94 \mathrm{fm}(\hbar \omega=15-11 \mathrm{MeV})$, as shown in Figure 18. As $b$ varies so does the predicted shape of the form factor: the width of the peak becomes narrower, and the peak itself shifts to lower momentum values with increasing $b$. The change in slope of the $\mathrm{C} 2$ matrix element with $b$ (Figure 18b) suggests that for sufficiently small $b$, the slope of the C2 matrix element will become negative, qualitatively agreeing with experiment. But such a small value of $b$ would would likely result in very slow convergence of the
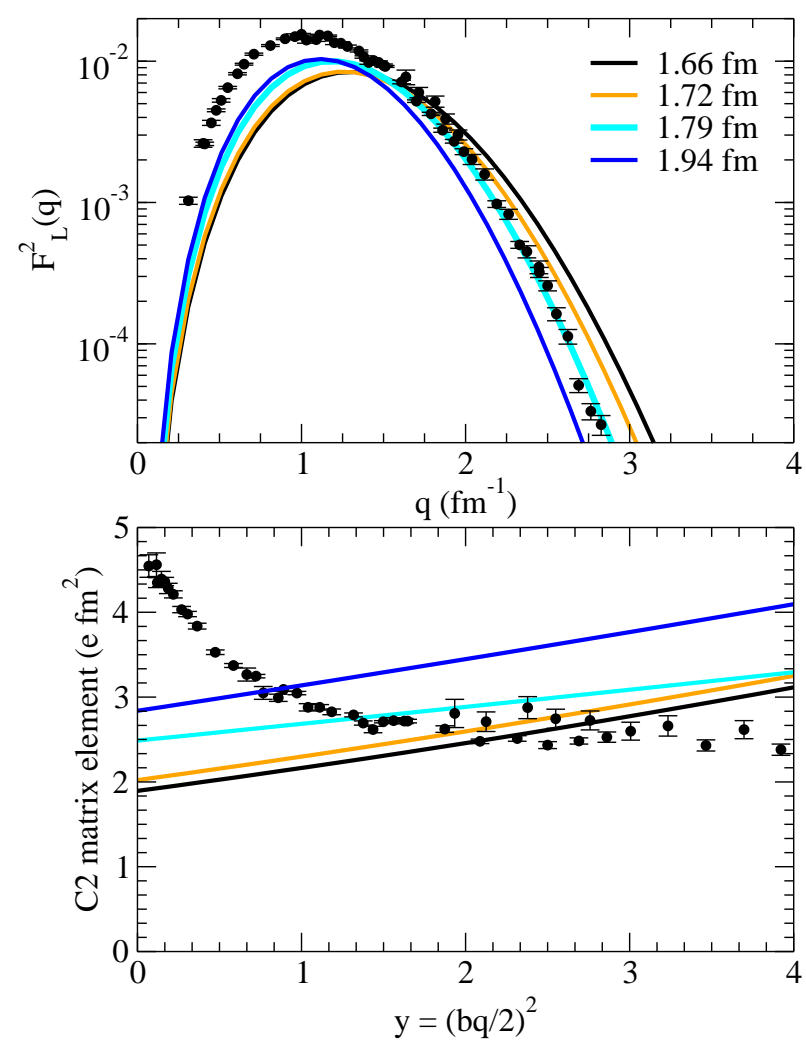

FIG. 18: (Color online) The charge form factor and the C2 matrix element for ${ }^{6} \mathrm{Li}$ are calculated for several values of $b$ in the $2 \hbar \omega$ model space.

calculations.

\section{B. Dependence of the ${ }^{12} \mathrm{C}$ Form Factor on the Oscillator Parameter}

In Figure 19 we display the dependence of the predicted $\mathrm{C} 2$ matrix element in ${ }^{12} \mathrm{C}$ on the oscillator parameter. These calculations were restricted to a $(0+2) \hbar \omega$ model space. As the oscillator parameter is varied, the value of $\langle p h|T+V| 0 \hbar \omega\rangle$ changes considerably and eventually changes signs. For sufficiently small $b(<1.33 \mathrm{fm})$ the slope of $\mathrm{C} 2(q)$ becomes negative, in qualitative agreement with experiment.

\section{Dependence of the Giant Resonances on the Oscillator Parameter}

Hoshino et al. 32] have pointed out that the problem with the sign of matrix elements of $\langle T+V\rangle$ in multi$\hbar \omega$ shell model calculations also manifests itself in the 


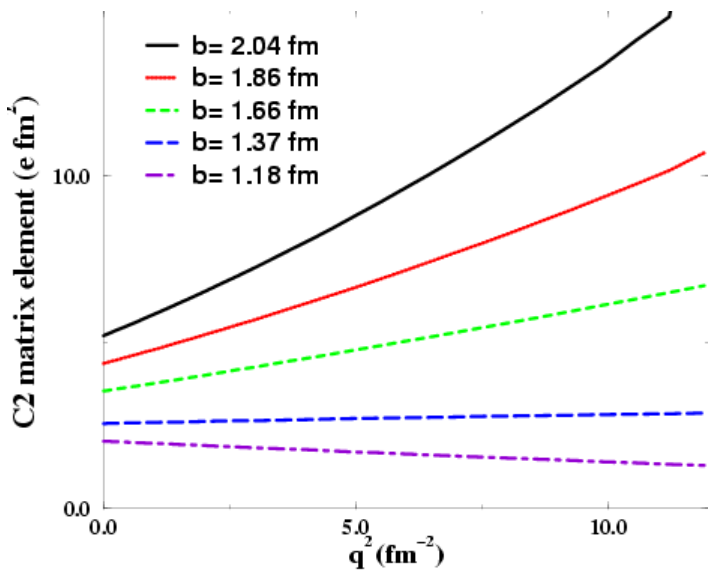

FIG. 19: (color online) Dependence of the C2 matrix element for the $4.44 \mathrm{MeV}$ state in ${ }^{12} \mathrm{C}$ on the oscillator parameter for the $(0+2) \hbar \omega$ model space.

predicted excitation of the GQR and GMR. The excitation energy of the GMR reflects the compressibility of the nucleus. Both are intrinsic properties of the nucleonnucleon interaction; and, the excitation energy should not depend on the properties of the $\mathrm{HO}$ well. In Figure 20 we show the predicted E0 strength for two different values of the oscillator parameter for our $(0+2) \hbar \omega$ model space. The large shift in the predicted excitation of the GMR from $\approx 35 \mathrm{MeV}$ to $\approx 60 \mathrm{MeV}$ occurs because of the change in the off-diagonal $\langle p h|T+V| 0 \hbar \omega\rangle$ matrix elements for the two values of the oscillator parameter. An analogous problem is seen with the E2 strength, Figure 21. We note that the sensitivity of the excitation energy of the giant resonances to the oscillator parameter would likely be considerably less for larger model spaces. But our $(0+2) \hbar \omega$ model space calculations exhibit similar sensitivity to that seen by Hoshino et al. [32]. As in the case of the $\left(e, e^{\prime}\right)$ form factors, the problem can be directly traced to the $\Delta \hbar \omega=2,(\lambda, \mu)=(2,0) p h$ excitations.

\section{Effect of an effective two-body electron scattering operator}

Since the model space sizes discussed here are finite, it is important to address the issue of the impact of effective $\left(e, e^{\prime}\right)$ operators and whether they can correct for the sign of the $\Delta \hbar \omega=2 p h$ excitations in the wave functions. Stetcu et al. 34] investigated how a two-body effective contribution affects the $\mathrm{E} 2$ and $\mathrm{C} 2$ operators. In the $0 \hbar \omega$ space, they found that the two-body operator moves the form factor in the same direction as the larger $(0+2) \hbar \omega$ calculations move; that is, the two-body operator results in the same unphysical enhancement of the form factor at large $q$.

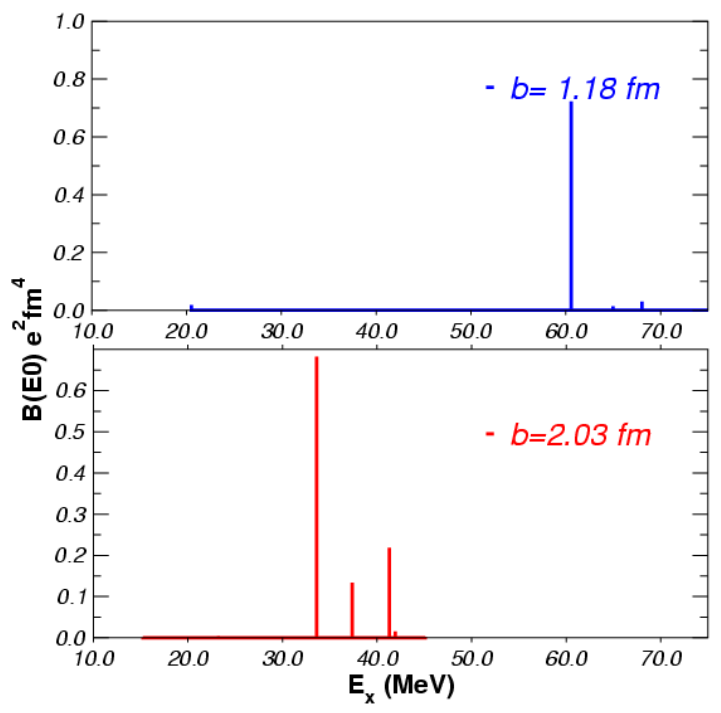

FIG. 20: Dependence of the predicted Giant Monopole Strength $(\mathrm{B}(\mathrm{E} 0))$ in ${ }^{12} \mathrm{C}$ on the oscillator parameter for the $(0+2) \hbar \omega$ model space.

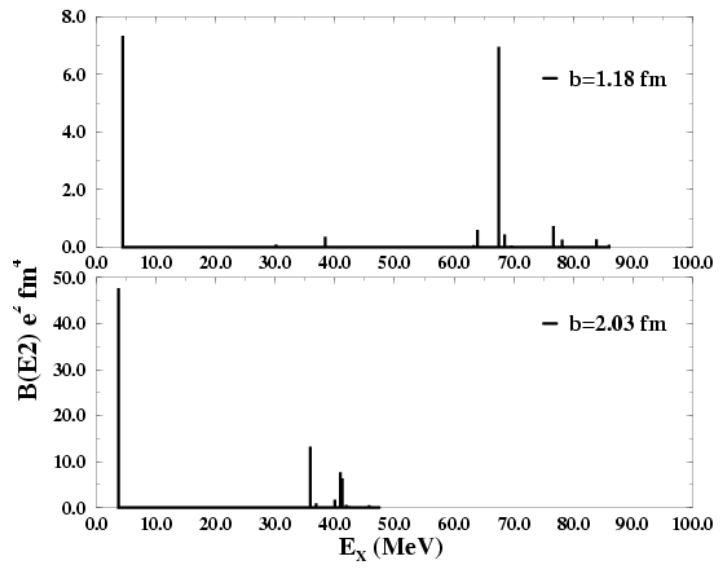

FIG. 21: Dependence of the $\mathrm{T}=0 \mathrm{E} 2$ strength built on the ground state of ${ }^{12} \mathrm{C}$ on the oscillator parameter for the $(0+$ 2) $\hbar \omega$ model space.

\section{CONCLUSION}

We have calculated the elastic $\mathrm{C} 0$ and the first excited state $\mathrm{C} 2$ charge form factors and the corresponding $\mathrm{C} 2$ matrix elements in ${ }^{6} \mathrm{Li}$ and ${ }^{12} \mathrm{C}$ within the NCSM using one-body bare operators. These calculations reveal two primary findings. First, the magnitude and sign of higher shell $p h$ amplitudes in the wavefunction do not behave as expected. Higher shell contributions add destructively at low $q$ and constructively at high $q$ to the form factors, contrary to experimental and theoretical expectations. The relative sign of the symplectic $(\lambda, \mu)=(2,0)$, $\Delta \hbar \omega=2$ amplitudes cause them to add destructively to the charge radii. The large $0 s \rightarrow n s$ (and $0 p \rightarrow n p$ for ${ }^{12} \mathrm{C}$ ) amplitudes, introduced by the Lee-Suzuki transfor- 
mation, also affect the shape of the form factors, further increasing the magnitude of the form factors at high momentum transfers. In the larger model spaces we explored for ${ }^{6} \mathrm{Li}$, the sign of the $2 \hbar \omega$ contributions to the inelastic form factor changes, but convergence onto experiment is slow.

The second main finding is the strong dependence of the magnitude and sign of the off diagonal $\Delta \hbar \omega=2$ matrix elements of $T+V$ on the oscillator parameter. As a result, the observables (the $\mathrm{C} 2$ form factor, GMR, GQR) also heavily depend on the choice of oscillator parameter. Furthermore, the $p h$ configurations in the low lying wave functions appear with an unexpected sign. These results indicates a lack of self-consistency in the NCSM similar to that found in the standard HO shell model. While there is no known solution, the effects may be minimized by including a Hartree-Fock condition in the calculations A prescription [28, 32] used in HO shell model calculations is to invoke the following condition:

$$
\langle(n+2) \hbar \omega p h|T+V| n \hbar \omega\rangle^{(\lambda, \mu)=(2,0)}=0
$$

Certainly this method bears further investigation. But in any case a correct treatment of the symplectic terms in the wave functions is crucial to obtaining a realistic description of electron scattering form factors within a $\mathrm{HO}$ shell model basis [35]. Invoking eq. (7) and/or another prescription to handle the $\Delta \hbar \omega=2$ and higher $p h$ excitations may lead the NCSM to have as much success in predicting momentum-based observables as in predicting energy spectra.

\section{Acknowledgments}

We wish to thank Petr Navrátil for providing us with the NCSM one-body density matrix elements used in these studies, and for many detailed and valuable discussions. We also wish to thank Ingo Sick, Gerry Peterson and Hall Crannell for providing us with their unpublished electron scattering data.
[1] P. Navrátil, J.P. Vary, W.E. Ormand, and B.R. Barrett, Phys. Rev. Lett. 87, 172502 (2001).

[2] P. Navrátil, J.P. Vary, B.R. Barrett, Phys. Rev. Lett. 84, 5728 (2000).

[3] P. Navrátil, J.P. Vary, W.E. Ormand, and B.R. Barrett, Phys. Rev. C 62, 054311 (2000).

[4] C. Forssen, E. Caurier, P. Navratil, arXiv:0901.0453v1 [nucl-th], (2009).

[5] S.Y. Lee and K. Suzuki, Phys. Lett. B 91 (1980) 173; K. Suzuki and S.Y. Lee, Prog. Theor. Phys. 64 (1980) 2091.

[6] A.C. Hayes, P. Navrátil, and J.P. Vary, Phys. Rev. Lett. 99, 012502 (2003).

[7] Tomas Dytrych, Kristina C Sviratcheva, Chairul Bahri, Jerry P. Draayer, James P. Vary, Phys. Rev. C 98, 162503 (2007).

[8] J. Carlson and R. Schiavilla, Rev. Mod. Phys. 70, 743 (1998).

[9] J.C. Bergstrom, Nucl. Phys. A327, 458 (1979).

[10] R.M. Hutcheon and H.S. Caplan, Nucl. Phys. A127, 417 (1969).

[11] R. Yen, L.S. Cardman, D. Kalinsky, J.R. Legg, and C.K. Bockelman, Nucl. Phys. A235, 135 (1974).

[12] G.C. Li, I. Sick, R.R. Whitney, and M.R. Yearian, Nucl. Phys. A162, 583 (1971).

[13] J.C. Bergstrom and E.L. Tomusiak, Nucl. Phys. A262, 196 (1976).

[14] J.C. Bergstrom, U. Deutschmann, R. Neuhausen, Nucl. Phys. A327, 439 (1979).

[15] R. Machleidt, F. Sammarruca, and Y. Song, Phys. Rev. C 53, 1483 (1996).

[16] R.B. Wiringa and R. Schiavilla, Phys. Rev. Lett. 81, 4317 (1998).
[17] H. L. Crannell and T.A. Griffy, Phys. Rev. 136 (1964) B1580

[18] Hall Crannell, Phys. Rev. 148 (1966) 1107

[19] A. Nakada, Y. Torizuka, Y. Horikawa, Phys. Rev. Lett. 27 (1971) 745

[20] Ingo Sick, private commutation

[21] D.J. Millener, D.I. Sober, H. Crannell, J.T. O'Brien, L.W. Fagg, S. Kowalski, C.F. Williams, and L. Lapikas, Phys. Rev. C 39, 14 (1989).

[22] G.G. Simon, Ch. Schmitt, F. Borkowski,, and V.H. Walther, Nucl. Phys. A333 (1980) 381

[23] L.J. Tassie and F.C. Barker, Phys. Rev. 111, 940 (1958).

[24] B. S. Pudliner et al. Phys. Rev. C 56, 1720 (1997).

[25] S. A. Coon and H. K. Han, Few-Body Systems 30, 131 (2001).

[26] S.S.M. Wong, Phys. Lett. 20, 188 (1966).

[27] M.W. Kirson, Nucl. Phys. A257, 58 (1976).

[28] A.C. Hayes, J.L. Friar. and D. Strottman, Phys. Rev. C 41, 1727 (1990).

[29] E.K. Warburton, J.A. Becker, and B.A Brown, Phys. Rev. C41, 1147 (1990).

[30] W.C. Haxton and C. Johnson, Phys. Rev. Lett. 65, 1325 (1990).

[31] J.P. Blaizot, Phys. Rep. 64, 1 (1980).

[32] Tsutomu Hoshino, Hiroyuki Sagawa and Akito Arima, Nucl. Phys. A481, 458 (1988).

[33] D.J. Millener, A.C. Hayes, and D. Strottman, Phys. Rev C 45, 473 (1992).

[34] Ionel Stetcu, Bruce R. Barrett, Petr Navratil, and James P. Vary, Phys. Rev. C. 71044325 (2005), and Ionel Stetcu private communication.

[35] Jutta Escher, J. Phys. G 25 783-786, (1999). 